\title{
Coalescence Behavior of Water Nanoclusters: Temperature and Size Effects
}

\author{
Ming-Liang Liao* \\ Department of Aircraft Engineering, Air Force Institute of Technology, Kaohsiung, Taiwan, Republic of China
}

\begin{abstract}
Shin-Pon Ju* and Sheng-Hui Yang
Department of Mechanical and Electro-Mechanical Engineering, Center for Nanoscience and Nanotechnology, National Sun Yat-Sen University, Kaohsiung, Taiwan, Republic of China
\end{abstract}

Received: September 24, 2006; In Final Form: March 21, 2007

\begin{abstract}
Temperature and size effects on coalescence behavior of water nanoclusters are investigated in this paper by means of molecular dynamics simulations. The flexible three-centered (F3C) water model is employed in the molecular dynamics simulations. To discuss the coalescence behavior, the whole coalescence process is separated into three stages, including the approaching stage, the coalescing stage, and the coalesced stage, according to the shrinkage evolution of the coalescence process. It is observed from the present study that, with a higher initial temperature and a smaller cluster size, the coalescence rate will amplify. The temperature and temperature fluctuation are highest at the coalescing stage, and the fluctuation is more apparent for a higher initial temperature and a smaller cluster size. Moreover, the average number of hydrogen bonds per water molecule will grow slightly after the coalescence of the water nanoclusters. Variations in the average number of hydrogen bonds during the coalescence increase for a higher initial temperature and a smaller cluster size.
\end{abstract}

\section{Introduction}

Coalescence of fluid droplets plays an important role in many physical, chemical, and biochemical processes. Several studies associated with these processes have been accomplished, such as collision of water clusters, ${ }^{1}$ vapor deposition on solid substrates, ${ }^{2}$ fuel combustion characteristics, ${ }^{3}$ and microfluidic biochemical reactions. ${ }^{4}$ Recently, with rapid advancements in nanotechnology, research on collision and coalescence of liquid nanodroplets has attracted much attention. Both experimental and numerical simulation methods have been used in the investigation of such phenomena. In regards to experimental methods, for example, Sing et al. ${ }^{5}$ utilized the light-scattering technique to observe interactions and the coalescence of nanodroplets in translucent $\mathrm{O} / \mathrm{W}$ (oil in water) emulsions. They found that the coalescence rates of the nanodroplets are slowed down when the surfactant molecular weight is high. Although there is some achievement in experimental study of the coalescence of liquid nanodroplets, most of the experimental works have focused on viscous systems. Coalescence rates of water nanodroplets (an inviscid fluid) are very difficult to measure owing to the rapid movements of the water droplets. ${ }^{6}$

With concern for numerical simulation methods, the molecular dynamics (MD) simulation method is a very popular approach since, with proper potentials for intermolecular interactions, this method can afford fairly accurate results, especially for dynamic response at the molecular level. Several studies concerning collision dynamics of water nanodroplets have been achieved by means of molecular dynamics simulations. For instance, Greenspan and $\mathrm{Heath}^{7}$ simulated the morphology evolution of two colliding water nanodroplets. A very simple molecular model was employed in their work, in

* To whom correspondence should be addressed. E-mail: mlliao@ mail.afats.khc.edu.tw (M.-L.L.); jushin-pon@mail.nsysu.edu.tw (S.-P.J.). which each water molecule was modeled as a single particle and a Lennard-Jones potential was applied to model the intermolecular interactions of the water molecules. Several fundamental collision modes were illustrated for different initial velocities specified on the colliding nanodroplets. A similar study was also performed later by Svanberg et al. ${ }^{1}$ to investigate collision dynamics of large water clusters. The rigid simple point charge (SPC) model was utilized to model individual water molecules. With the examples of large clusters' collision, they concluded that nanoclusters in some respects resemble macroscopic objects.

In regards to molecular dynamics simulations for coalescence (the collision without initial relative velocity) of liquid nanodroplets, Koplik and Banavar ${ }^{8}$ explored the coalescence of two liquid nanodroplets in shear flow induced by another immiscible fluid. Both a simple molecular model and a Lennard-Jones potential were utilized in their study. They discovered that the coalescence starts when molecules near the boundary of one nanodroplet thermally fluctuate into the range of attraction of the other nanodroplet and then form a string of mutually attracting molecules, i.e., a bridge for further coalescing. Recently, Zhao and $\mathrm{Choi}^{9}$ have studied the coalescence of two water nanodroplets in $n$-heptane. Coalescence of two water nanodroplets in vacuum was also examined in their research. A modified DREIDING 2.21 force field $^{10}$ in a commercial software package CERIUS2 was used to model the water molecules. Snapshots from the simulations for coalescence of the water nanodroplets were displayed in the research. They revealed that the coalescence rate of water nanodroplets in $n$-heptane is considerably slower than that in vacuum, especially in the first $10 \mathrm{ps}$ of the coalescence process.

Although the above results can give some insights into the coalescence of water nanodroplets, more detailed behavior of the water nanodroplets during the whole coalescence process 
seems to be undeveloped, especially in terms of the effects of temperature and size on the coalescence behavior. In this paper, the effects of temperature and size on the coalescence behavior of two water nanoclusters were explored. Properties of the water nanoclusters during the whole coalescence process, such as shrinkage evolution, temperature variations, and variations in the average number of hydrogen bonds per water molecule, were examined by the use of molecular dynamics simulations. The flexible three-centered (F3C) water model ${ }^{11}$ employed fairly well in our previous works ${ }^{12,13}$ was also utilized in the present research. To explore the temperature and size effects, two identical nominally spherical water nanoclusters of various sizes at different initial temperatures were selected in this examination.

\section{Simulation Method}

Molecular dynamics simulations were utilized in the present research. In the simulations, the $\mathrm{F} 3 \mathrm{C}$ water model well applied in our previous works ${ }^{12,13}$ was also employed in the present study to model the inter- and intramolecular interactions of water molecules in the water nanoclusters. The water model is outlined below. The $\mathrm{F} 3 \mathrm{C}$ water potential $U$ can be expressed as ${ }^{11}$

$$
U=U_{\text {bond }}+U_{\text {bend }}+U_{\mathrm{vdw}}+U_{\mathrm{els}}
$$

where potentials $U_{\text {bond }}, U_{\text {bend }}, U_{\mathrm{vdw}}$, and $U_{\text {els }}$ represent the bond strength energy, the bending energy, the van der Waals potential, and the electrostatic potential of the water molecules, respectively. The former two potentials are written as

$$
\begin{gathered}
U_{\text {bond }}=\sum K_{\mathrm{b}}^{\mathrm{OH}}\left(b_{i}-b_{0}^{\mathrm{OH}}\right)^{2} \\
U_{\text {bend }}=\sum K_{\theta}^{\mathrm{HOH}}\left(\theta_{i}-\theta_{0}^{\mathrm{HOH}}\right)^{2}
\end{gathered}
$$

where $b_{i}, b_{0}^{\mathrm{OH}}, \theta_{i}$, and $\theta_{0}^{\mathrm{HOH}}$ are the $i$ th $\mathrm{O}-\mathrm{H}$ bond length, the equilibrium length of the $\mathrm{O}-\mathrm{H}$ bond, the $i$ th $\mathrm{H}-\mathrm{O}-\mathrm{H}$ bending angle, and the equilibrium angle of the $\mathrm{H}-\mathrm{O}-\mathrm{H}$ bending angle of the water molecule, respectively. The summations in eqs 2 and 3 are performed over all bond lengths and bond angles in the water molecules, respectively.

The van der Waals potential $\left(U_{\mathrm{vdw}}\right)$ added in eq 1 is to model the intermolecular energy of the water molecules. For nonbonded pairs $i$ and $j$ in the water molecules with a distance $r_{i j}$, the van der Waals potential can be expressed as

$$
U_{\mathrm{vdw}}=\sum\left[A_{\mathrm{sc}} \epsilon^{i j}\left(r_{0}^{i j} / r_{i j}\right)^{12}-2 \epsilon^{i j}\left(r_{0}^{i j} / r_{i j}\right)^{6}-S_{\mathrm{vdw}}\left(r_{i j}\right)\right]
$$

where the parameter $A_{\mathrm{sc}}$ is utilized to compensate for the interaction lost for small cutoff distances. The energy parameter $\epsilon^{i j}$, the distance parameter $r_{0}^{i j}$, and the truncation shift function $S_{\mathrm{vdw}}$ in the van der Waals potential can be attained from ref 11 .

In eq 1 the electrostatic potential, $U_{\mathrm{els}}$, for atoms $i$ and $j$ in the water molecules is represented by

$$
U_{\mathrm{els}}=\sum\left[q^{i} q^{j} / r_{i j}-S_{\mathrm{els}}\left(r_{i j}\right)\right]
$$

where $q^{i}$ and $q^{i}$ are the partial charges of the hydrogen or oxygen atoms of two water molecules within the cutoff distance. The truncation shift function $S_{\text {els }}$ for the electrostatic potential is also provided in ref 11 . The summations in eqs 4 and 5 are carried out over all nonbonded pairs $i$ and $j$ closer than the cutoff distance. All the F3C parameters selected in the present research can be found in our previous work. ${ }^{12}$

To examine of the coalescence behavior of water nanoclusters, two identical spherical water nanoclusters each consisting of $N$

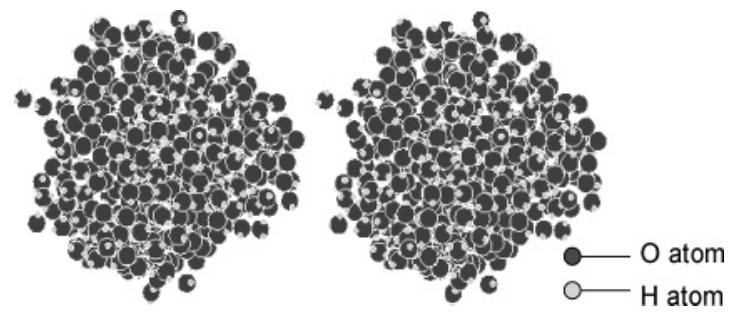

Figure 1. Schematic diagram of the simulation model (initial configuration of the coalescence system).

water $\left(\mathrm{H}_{2} \mathrm{O}\right)$ molecules were used in the simulations. Three different cluster sizes (with $N=195,446$, and 803) and three distinct initial temperature conditions (with $T=273,300$, and $320 \mathrm{~K}$ ) were selected to investigate the temperature and size effects on the coalescence behavior of the water nanoclusters. At the beginning, a single spherical water model composed of $N$ water molecules was generated by duplication from a unit water cell. The velocities of all atoms in the single water model were then randomly assigned according to a Maxwell distribution for temperature of $1 \mathrm{~K}$ by the Nosé-Hoover method. ${ }^{14,15}$ Molecular dynamics simulations were then performed in an NVT (canonical) ensemble. In the simulations, the velocity Verlet's algorithm ${ }^{14,15}$ was invoked for time integration of Newton's equations of motion to obtain the new velocities and positions of each atom. Furthermore, a time step of $10^{-15} \mathrm{~s}$ ( $1 \mathrm{fs})$ was employed during the time integration. For simulation stability, 5000 initial iterations for system equilibrium were executed first, and then the temperature was raised gradually from $1 \mathrm{~K}$. The simulations continued until the system achieved the desired temperature (i.e., $T=273$, 300, or $320 \mathrm{~K}$ ). Under this temperature, the simulations lasted until equilibrium in energy had been obtained.

With this single water nanocluster in equilibrium, duplication of the water nanocluster was made to gain the other counterpart with identical states. The two identical water nanoclusters were then deposited in vacuum at a distance $3 \AA$ from each other (as shown in Figure 1) for the coalescence simulations. Selection of the initial separation distance between the coalescing nanoclusters is not critical for the coalescence simulations. Any distance small enough to be within the interaction range of the intermolecular forces is suitable for the simulations. In the present study, the value of $3 \AA$ employed by Zhao et al. ${ }^{9}$ for coalescence of water droplets in vacuum was also invoked in the simulations. It is noted that this value was also appropriate for coalescence (or sintering) analysis of metal nanoparticles using molecular dynamics simulations. For instance, Raut et al. ${ }^{16}$ has investigated sintering of aluminum nanoparticles, and Arcidiacono et al. ${ }^{17}$ examined the coalescence of gold nanoparticles.

In the current research, during the coalescence simulations an NVE (microcanonical) ensemble was utilized rather than an NVT (canonical) ensemble. We did so because most coalescence experiments were performed under a vacuum environment. The heat released in the coalescence cannot be taken away very effectively. This will cause a temperature rise during the coalescence. Thus, the NVE ensemble can afford a more realistic model for the coalescence system. Since the present coalescence simulations were also carried out in vacuum conditions, the NVE ensemble was chosen during the coalescence. It is noticed that the NVE ensemble was also used by many researchers for sintering/coalescence simulations of nanoparticles. ${ }^{16-19}$ Specifically, in the study of Raut et al. ${ }^{16}$ some simulations were also 


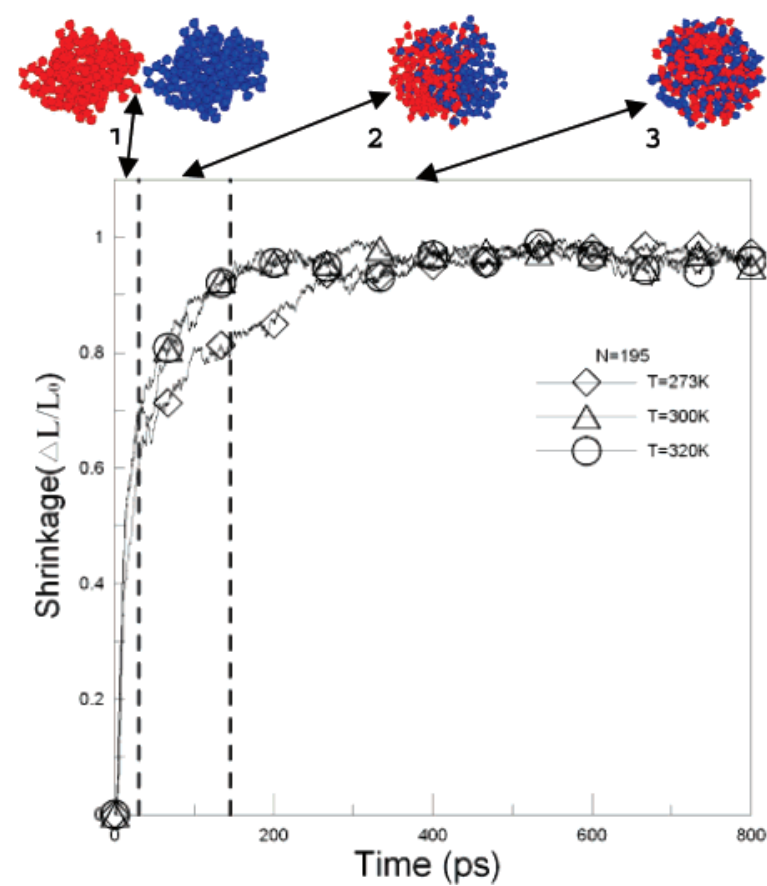

Figure 2. Shrinkage evolution during the coalescence of two water nanoclusters with $N=195$ at three different initial temperatures. For ease of illustration, the vertical dashed lines drawn for $T=300 \mathrm{~K}$ are used to designate the three stages of the coalescence process.

accomplished by the NVT ensemble in addition to their NVE ensemble simulations and the same qualitative results were attained.

With the initial configuration of the coalescence system, the NVE molecular dynamics simulations were performed for $1 \mathrm{~ns}$ (1000 ps). Data from the simulations was collected and averaged for the examination of temperature and size effects on the coalescence behavior of the two water nanoclusters.

\section{Results and Discussion}

The coalescence of two water nanoclusters with $N=195$ at three different initial temperatures of 273 , 300, and $320 \mathrm{~K}$ were first investigated in this study. Here, we used shrinkage evolution of the coalescing nanoclusters to track the coalescence process of the system. Figure 2 illustrates the shrinkage evolution during the coalescence. The value of shrinkage is calculated from the following equation

$$
\text { shrinkage }=\Delta L / L_{0}=\left(L_{0}-L\right) / L_{0}
$$

where $L$ and $L_{0}$ represent the distances between the mass centers of the two water nanoclusters during the coalescence and at the initial configuration, respectively. Initially, the two nanoclusters in equilibrium were placed with their center-to-center distance at $L_{0}=2 R+3 \AA$ (as stated previously and depicted in Figure 1), where $R$ denotes the radius of the nanoclusters and the separation distance ( $3 \AA$ ) is selected the same as that utilized in related studies. $7,9,16,17$ Thus at the beginning of the coalescence, $L$ equals to $L_{0}$ and the value of shrinkage is zero. As the nanoclusters start to coalesce, $L$ becomes less than $L_{0}$ and the value of shrinkage grows from zero. After the coalescence completes, the distance between the mass centers of the water nanoclusters, $L$, keeps constant and approximately approaches zero. As a consequence, the value of shrinkage also nearly approaches a constant value of 1.0. Hence, the value of shrinkage can be used to monitor when two nanoclusters will completely coalesce to one larger nanocluster. According to the shrinkage evolution in Figure 2, the whole coalescence process can be divided into three stages: Stage 1, the approaching stage; Stage 2, the coalescing stage; Stage 3, the coalesced stage. For ease of illustration, the shrinkage evolution at $300 \mathrm{~K}$ is selected to designate these three stages, which are separated by two vertical dashed lines in the figure. In addition, the corresponding morphology of the nanoclusters for these three stages is also appended at the top of the figure.

It is observed that at Stage 1 (i.e., the approaching stage) the two nanoclusters approach each other by the strong attractive forces between the nanoclusters and then the water molecules of one nanocluster penetrate into and further interact with the water molecules of the other nanoncluster at Stage 2 (i.e., the coalescing stage). At Stage 3 (i.e., the coalesced stage), the value of shrinkage is nearly constant, which means the accomplishment of coalescence. With the observation of Figure 2, it is evident that two water nanoclusters need a longer time to coalesce into a larger water nanocluster at a lower temperature. In our previous studies, ${ }^{12,13}$ we have investigated the temperature and size effects on the behavior of interior and surface water molecules of a water nanocluster. From the results of those studies, it is noticed that at higher temperatures the water nanoclusters have a larger ratio of surface water molecules, ${ }^{12}$ which possess greater mobility than the interior water molecular, and also have a shorter hydrogen-bond relaxation time. ${ }^{13}$ Accordingly, a shorter time is required for the coalescence of two water nanoclusters at higher initial temperatures.

To observe configuration of the water nanoclusters during the coalescence, Figure 3 displays snapshots for morphology of the coalescence system calculated from the present simulations for $N=195$ and $T=300 \mathrm{~K}$ (initially). Before approaching, the initial configuration $(t=0)$ of the system is depicted in Figure 1. At the beginning of the approaching stage (Stage 1), at $t=1 \mathrm{ps}$ (Figure 3a), surface water molecules in the interaction region start to move toward each other resulting from the strong attractive forces between the nanoclusters. The surface molecules then play as a bridge to further attract surface and interior molecules from both nanoclusters and result in initiation of the coalescence. At $t=10 \mathrm{ps}$ (Figure $3 \mathrm{~b}$ ), the initial gap ( $3 \AA$ ) between the nanoclusters is entirely filled by the water molecules and a neck is generated at the onset of the coalescence. Entering into the coalescing stage (Stage 2), at $t=50 \mathrm{ps}$ (Figure $3 \mathrm{c}$ ) the water molecules of one nanocluster penetrate into those of the other nanocluster and a merged single nanocluster having a near oval shape is developed. The interpenetration continues during the coalescing stage and at $t=100 \mathrm{ps}$ (Figure 3d) the merged nanocluster has an outline close to that of a sphere. Finally, the coalescence has completed at $t=200 \mathrm{ps}$ (Figure 3e). At this coalesced stage (Stage 3), water molecules of the two nanoclusters are thoroughly fused and a single spherical water nanocluster consisting of 390 water molecules is therefore attained. It is observed that the coalescence of the two water nanoclusters is very rapid (completing in 200 ps for the case of $N=195$ and $T=300 \mathrm{~K}$ ). In addition, the obtained morphology is found to be similar to that of Koplik and Banavar ${ }^{8}$ as well as Zhao and Choi. ${ }^{9}$

Since arrangements of water molecules are quite different at the three coalescence stages of two water nanoclusters, temperature variations as well as the standard deviation of the temperature at the three stages are noteworthy and are examined in Figure 4. For each temperature profile, the average temperatures at Stage 2 (the coalescing stage) are highest and the average temperatures at Stage 3 (the coalesced stage) are higher than those at Stage 1 (the approaching stage). This is because 


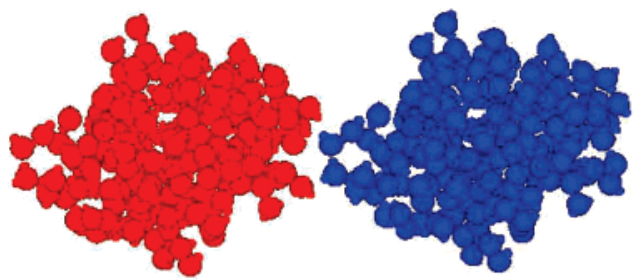

(a)

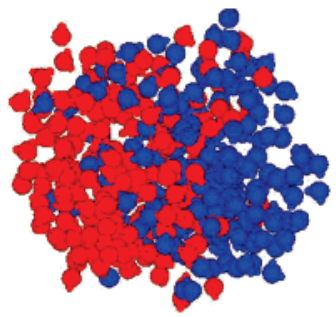

(c)

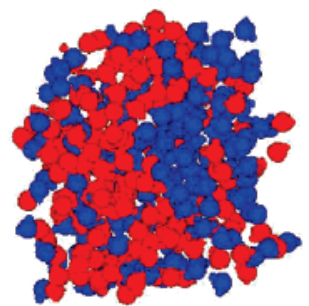

(d)

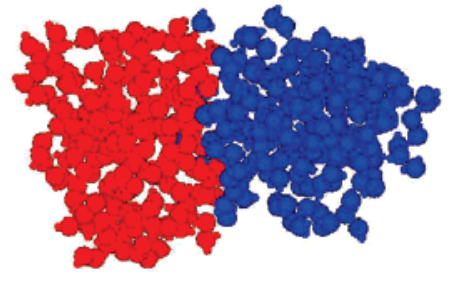

(b)

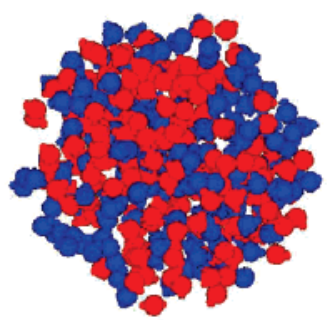

(e)

Figure 3. Snapshots for morphology of the coalescence system with $N=195$ and $T=300 \mathrm{~K}$. (a) $t=1 \mathrm{ps}$; (b) $t=10 \mathrm{ps;} \mathrm{(c)} t=50 \mathrm{ps}$; (d) $t=$ $100 \mathrm{ps}$; (e) $t=200 \mathrm{ps}$.

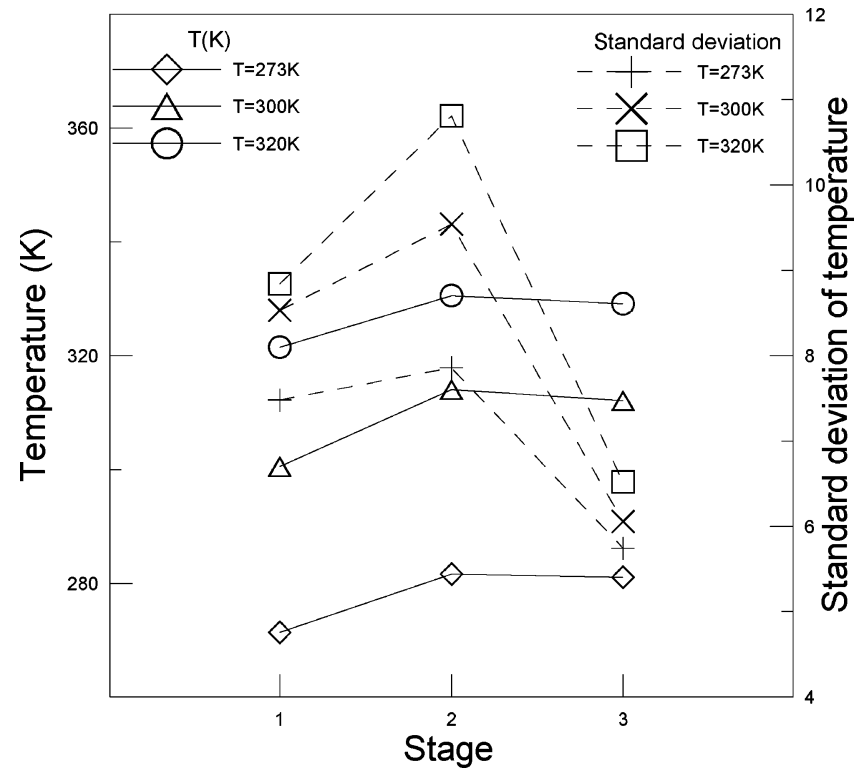

Figure 4. Temperature variations and the standard deviation of the temperature at the three coalescence stages for $N=195$. The solid and dashed lines are added for clarity.

as the two water nanoclusters start to coalesce, forming a larger nanocluster, new chemical bonds between the coalescing particles will build. To grow a more stable configuration, the particles will diminish their surface energy in order to reduce of their surface area. Since the coalescence occurs in a vacuum environment, the release surface energy will accelerate the thermal motion of the coalescing particles and simultaneously heat the water molecules owing to the conservation of total energy in an NVE ensemble. This energy transfer enhances during the coalescing stage (Stage 2). Therefore, the average temperatures are highest at Stage 2. Also, because the resulting heat cannot be carried away very effectively in the vacuum environment, the average temperatures of the nanoclusters will rise at the end of the coalescence (Stage 3). The temperature growth behavior during coalescence of water nanoclusters resembles the behavior of nanoparticles during sintering or coalescence. ${ }^{16-19}$ It is also observed from Figure 4 that the temperature fluctuation at Stage 2 is largest for various initial temperatures and enlarges at a greater initial temperature. The explanation is stated as follows. At the coalescing stage (Stage 2), the water molecules are accelerated resulting from the decrease of their surface energy. This intensifies the interpenetration of the coalescing water molecules and thus hastens the generation of the new chemical bonds as well as the energy transfer between the coalescing water molecules. The temperature fluctuation therefore is largest at this stage. Furthermore, since at a higher initial temperature the ratio of surface water molecules increases, ${ }^{12}$ the coalescing action will be further activated due to the higher mobility of the surface water molecules as compared with the interior water molecules. Thus, the temperature fluctuation heightens at a greater initial temperature. At Stage 3, however, the temperature fluctuation drops obviously, since the coalescence has finished at this stage.

Figure 5 shows variations of the average number of hydrogen bonds per water molecule of the water nanoclusters, $n_{\mathrm{HB}}$, at the three stages for the different initial temperatures. In determining the value of $n_{\mathrm{HB}}$, the geometric definition widely used in the related studies ${ }^{13,20,21}$ was also utilized in the present research. The geometric definition declares that two water molecules are claimed to be hydrogen bonded if their interoxygen distance is less than $3.5 \AA$ and the hydrogen-oxygen distance from the two molecules is less than $2.45 \AA$ and at the same time the oxygen-oxygen-hydrogen angle is less than a cutoff value of $30^{\circ}$. From the figure it is noticed that for each case, $n_{\mathrm{HB}}$ at Stage 3 (after coalescence) is slightly greater than that at Stage 1 (before coalescence). This observation corresponds to the size effect on the average number of hydrogen bonds of a water nanocluster (i.e., a greater water nanocluster has a larger $n_{\mathrm{HB}}$ ), as illustrated in our previous study. ${ }^{12}$ Moreover, it is observed that there is a drop in $n_{\mathrm{HB}}$ at Stage 2 . This is because for this case with $N=195$ the surface water molecules increase at the coalescing stage (as discussed in detail later) and the surface molecules have a lower average number of hydrogen bonds than the interior molecules. ${ }^{12}$ It should also be noticed that the drop in $n_{\mathrm{HB}}$ enlarges at a higher initial temperature. Since the ratio of surface water molecules increases at a higher initial temperature, ${ }^{12}$ this further amplifies the drop of the average number of hydrogen bonds during coalescence. 


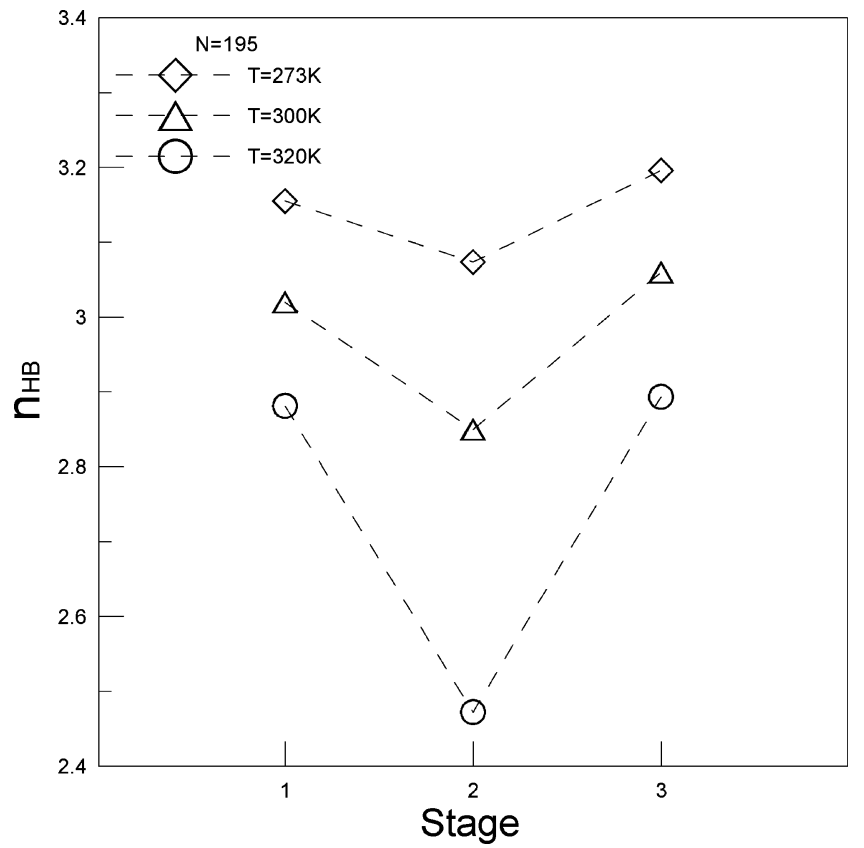

Figure 5. Average number of hydrogen bonds per water molecule, $n_{\mathrm{HB}}$, at the three coalescence stages for $N=195$ at the different initial temperatures.

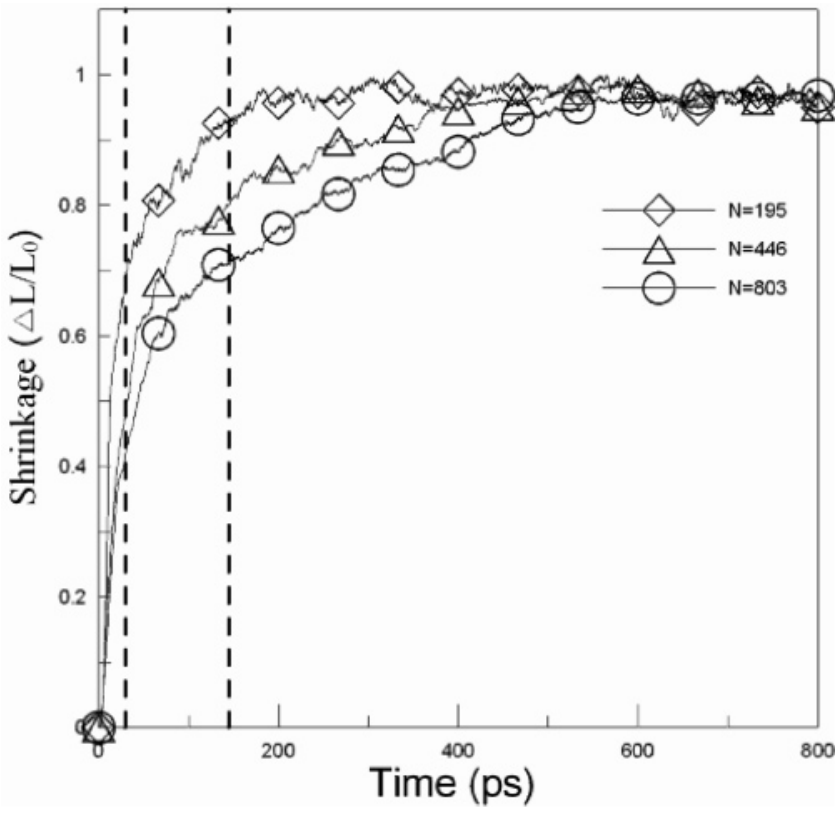

Figure 6. Shrinkage evolution during the coalescence of two water nanoclusters with $N=195,446$, and 803 at $300 \mathrm{~K}$ initially. For ease of illustration, the vertical dashed lines drawn for $N=195$ are used to designate the three stages of the coalescence process.

Effects of the cluster size on coalescence of two water nanoclusters could be observed from the shrinkage evolution in Figure 6. The coalescence times (i.e., times for achievement of coalescence) obtained from the simulations are about 150 , 450, and 540 ps for the system with $N=195,446$, and 803, respectively. It is evident that it requires a shorter time for the smaller water nanoclusters to finish the coalescence. This observation can also be elucidated by the ratio of surface water molecules of the coalescing nanoclusters. Since at the same temperature a smaller water nanocluster has a greater ratio of the surface molecules than a larger water nanocluster, ${ }^{12}$ the coalescence time for smaller water nanoclusters will be shorter

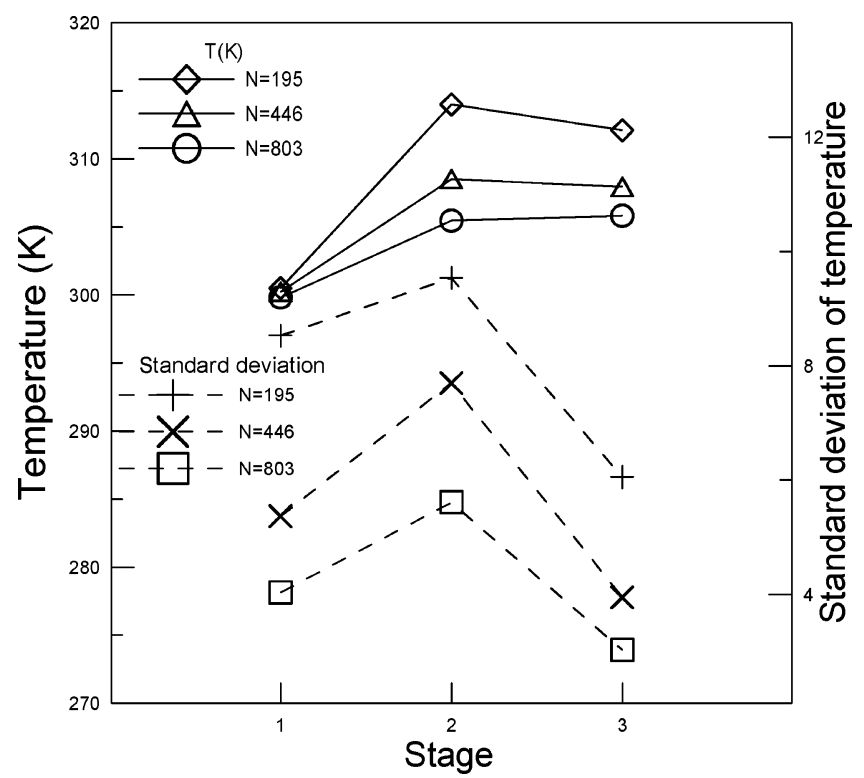

Figure 7. Temperature variations and the standard deviation of the temperature at the three coalescence stages for $N=195,446$, and 803 at $300 \mathrm{~K}$ initially.

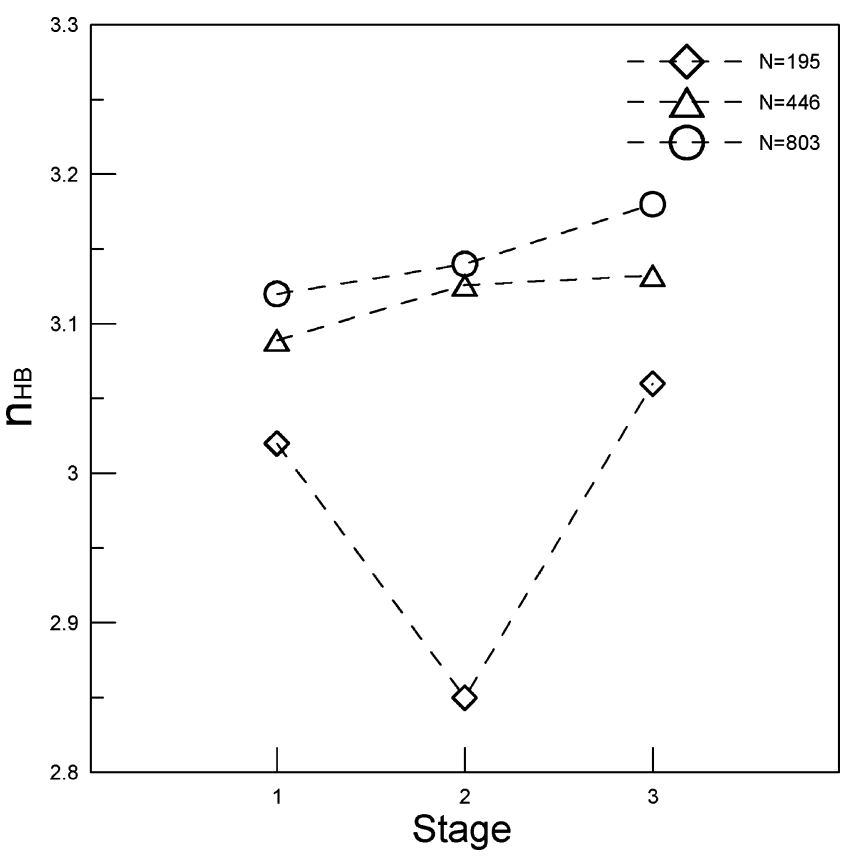

Figure 8. Average number of hydrogen bonds per water molecule, $n_{\mathrm{HB}}$, at the three coalescence stages for $N=195,446$, and 803 at $300 \mathrm{~K}$ initially.

than that for the larger ones owing to the higher mobility of the surface water molecules.

Figure 7 displays the temperature variations as well as the standard deviation of the temperature at the three coalescence stages for two water nanoclusters at $300 \mathrm{~K}$ (initially) with $N$ $=195,446$, and 803, respectively. Variations of the temperature fluctuation at the three stages are similar to those in Figure 4, which shows the largest temperature fluctuation at Stage 2 owing to the greatest energy transfer at this stage. With the same reasoning considered for size effects on the coalescence rate, the temperature rise during the coalescence is largest for the smallest water nanoclusters (with $N=195$ ). This observation can also be found during sintering of aluminum nanoparticles ${ }^{16}$ and silicon nanoparticles. ${ }^{18}$ 
Figure 8 illustrates the average number of hydrogen bonds per water molecule, $n_{\mathrm{HB}}$, at the three coalescence stages of two water nanoclusters at $300 \mathrm{~K}$ (initially) with $N=195,446$, and 803 , respectively. As expected, $n_{\mathrm{HB}}$ grows with the increase in size of the nanoclusters at the end of the coalescence. For the two larger cases $(N=446$ and 803$)$, the value of $n_{\mathrm{HB}}$ will slightly increase during the whole coalescence process, while for the smallest case the value will drop at Stage 2 and then increase at Stage 3 to a value higher than that of Stage 1. The drop of $n_{\mathrm{HB}}$ for the case of $N=195$ at Stage 2 can be attributed to the larger ratio of surface water molecules in this case. For $N=195$ the ratio of surface water molecules is 55\%. ${ }^{12}$ Namely, before coalescence the number of surface water molecules is over that of interior water molecules. Since the surface molecules have greater mobility than the interior molecules, the large amount of surface molecules will raise the coalescence rate and thus accelerate the interaction between the surface molecules and the interior molecules at Stage 2. This will yield more surface molecules and lead to an apparent increase in the number of surface water molecules. The value of $n_{\mathrm{HB}}$ is therefore reduced due to the rapid growth of the surface water molecules. This is because surface molecules have a lower average number of hydrogen bonds than the interior molecules. ${ }^{12}$ In addition, one should note that the drop in $n_{\mathrm{HB}}$ at Stage 2 also occurs for other initial temperature conditions, as depicted in Figure 5. The above surface effect is more pronounced as the size of the nanocluster is reduced. For $N=446$ and 803, however, the ratios of surface water molecules before coalescence are 45 and $42 \%$, respectively. ${ }^{12}$ The amount of surface molecules will not evidently increase at Stage 2 for these two cases. Hence, the drop in $n_{\mathrm{HB}}$ at Stage 2 will not appear.

\section{Conclusions}

Temperature and size effects on coalescence behavior of water nanoclusters have been examined with the use of molecular dynamics simulations. In the simulation model, the F3C water model was employed and two spherical water nanoclusters of equal size were used. For ease of discussing the coalescence behavior, the whole coalescence process is separated into three stages including Stage 1, the approaching stage; Stage 2, the coalescing stage; Stage 3, the coalesced stage, according to the shrinkage evolution of the coalescence process. The present study shows that at a higher initial temperature, since the water nanoclusters have a larger ratio of surface water molecules (having a higher mobility), the coalescence rate will heighten. During the coalescence, the temperature and temperature fluctuation are highest at Stage 2 (the coalescing stage), and the fluctuation is more obvious at a higher initial temperature. The explanation is due to the heat release resulting from the reduction of surface energy at the coalescing stage. Hence, the temperature rise and temperature fluctuation are most evident at Stage 2. Moreover, the average number of hydrogen bonds per water molecule will increase slightly after the coalescence of the water nanoclusters. Variations in the average number of hydrogen bonds during the coalescence amplify for a higher initial temperature condition.

Regarding size effects on coalescence behavior of water nanoclusters, it is observed that the coalescence rate grows with a small cluster size. During the coalescence, the temperature rise and temperature fluctuation are more manifest for water nanoclusters with a smaller cluster size. This is because a smaller water nanocluster has a larger ratio of surface molecules (i.e., the surface effect) and with the larger amount of surface molecules the energy transfer will augment. Therefore, it raises the heat release during the coalescence. Moreover, variations of the average number of hydrogen bonds per water molecule during the coalescence enlarge for small water nanoclusters due to the larger ratio of surface molecules in the smaller nanoclusters.

Although we have gained some insights into the coalescence behavior of water nanoclusters with the aid of the coalescence simulations of two identical water nanoclusters, there are certain topics still yet to be explored. The coalescence behavior of two water nanoclusters of different sizes is an interesting one. Besides the current temperature and size effects, the effect of cluster size ratio on the coalescence behavior should also be examined for an even more developed understanding. In addition, the coalescence behavior of multiple water nanoclusters (e.g., three or four nanoclusters) is another issue worthy of being investigated. Results from this problem would be beneficial to increase awareness of the multiple-nanocluster coalescence. Certain topics among these are the future work of the authors.

Acknowledgment. The authors gratefully acknowledge the support provided to this research by the National Science Council of the Republic of China under Grant No. NSC-095SAF-I-564-623-TMS.

\section{References and Notes}

(1) Svanberg, M.; Ming, L.; Markovic, N.; Pettersson, J. B. C. J. Chem Phys. 1998, 108, 5888.

(2) Chen, L. H.; Chen, C. Y.; Lee, Y. L. Surf. Sci. 1999, 429, 150.

(3) Wang, C. H.; Lin, C. Z.; Hung, W. G.; Huang, W. C.; Law, C. K. Combus. Sci. Tech. 2004, 176, 71.

(4) Curran, K.; Colin, S.; Baldas, L.; Davies, M. Microfluidics Nanofluidics 2005, 1, 336.

(5) Sing, A. J. F.; Graciaa, A.; Lachaise, J.; Brochette, P.; Salager, J. L. Colloids Surf. A 1999, 152, 31.

(6) Brinker, C. J.; Scherer, G. W. Sol-Gel Science; Academic: New York, 1990.

(7) Greenspan, D.; Heath, L. F. J. Phys. D 1991, 24, 2121.

(8) Koplik, J.; Banavar, J. R. Science 1992, 257, 1664.

(9) Zhao, L.; Choi, P. J. Chem. Phys. 2004, 120, 1935.

(10) Mayo, S. L.; Olafson, B. D.; Goddard, W. A., III. J. Phys. Chem. 1990, 94, 8897.

(11) Levitt, M.; Hirshberg, M.; Sharon, R.; Laidig, K. E.; Daggett, V. J. Phys. Chem. B 1997, 101, 5051.

(12) Ju, S. P.; Yang, S. H.; Liao, M. L. J. Phys. Chem. B 2006, 110, 9286.

(13) Ju, S. P.; Liao, M. L.; Yang, S. H.; Lee, W. J. Mol. Phys. 2007, $105,429$.

(14) Haile, J. M. Molecular Dynamics Simulation; Wiley-Interscience: New York, 1992.

(15) Rapaport, D. C. The Art of Molecular Dynamics Simulations; Cambridge University Press: Cambridge, UK, 2004.

(16) Raut, J. S.; Bhagat, R. B.; Fichthorn, K. A. Nanostruct. Mater. 1998, 10, 837 .

(17) Arcidiacono, S.; Bieri, N. R.; Poulikakos, D.; Grigoropoulos, C. P. Int. J. Multiphase Flow 2004, 30, 979.

(18) Zachariah, M. R.; Carrier, M. J. J. Aerosol Sci. 1999, 9, 1139.

(19) Hendy, S. Phy. Rev. B 2003, 68, 241403.

(20) Luzar, A. J. Chem. Phys. 2000, 113, 10663.

(21) Chandra, A. Phys. Rev. Lett. 2000, 85, 768 